\title{
A NEW VIDEO-OBJECT WATERMARKING SCHEME ROBUST TO OBJECT MANIPULATION
}

\author{
P. Bas and B. Macq* \\ Laboratoire Tele, Batiment Stevin, Place du Levant, \\ B-1348 Louvain-la-Neuve, Belgium
}

\begin{abstract}
This paper presents a watermarking scheme for image or video objects. The watermarking of video objects implies different constraints from raw watermarking methods. The mark has to be detected after object manipulations such as rotations, translations and VOL modifications. To achieve these requirements, the embedding scheme exploits the shape of the object: a random sequence is transformed to fit the scale and the orientation of the object. The detection of the mark is performed applying an inverse transform and calculating a correlation between the random sequence and the warped object. Our results illustrate the fact that the presented method is robust to object manipulation.
\end{abstract}

\section{INTRODUCTION}

With the emergence of new multimedia standards such as Mpeg-4 and Jpeg-2000, the notion of video-object or imageobject is more and more widespread [1]. Consequently, a need to protect the different objects of an image or a video appeared. The watermarking technologies enable the embedding of an unnoticeable and indelible mark inside multimedia contents such as images, video or audio [2]. It appears that the new multimedia standards are strongly bounded with watermarking methods. For example, the Mpeg-4 group designed an IPMP (Intellectual Property Management \& Protection) layer presenting a watermarking structure to permit monitoring, copy control and fingerprinting Mpeg-4 contents [3].

This paper is devoted to present a new video-object watermarking scheme that can be employed to protect standard allowing interaction with visual content and object manipulation.

\subsection{Video Object Watermarking Context}

Object watermarking is quite different from bit-stream or compressed watermarking of image or video. In the field of object watermarking, the embedded mark has to be detected after object manipulations such as:

\footnotetext{
* This research was supported by the European IST Aspis project
}

- The insertion of a marked Video Object (VO) in a set of other VO (named VOL in the Mpeg-4 context to denote Video Object Layer).

- A shifting operation applied to the marked VO inside the VOL.

- A rotation or a scaling of the VO.

Different approaches have been designed to enable Mpeg4 watermarking. Barni et al presented a method to mark Video Object Plans (VOP) [4]. The embedding of the mark is performed modifying selected pair of DCT quantized coefficients in each VOP. The selection of the marked macroblocks is performed using a pseudo-random sequence and consequently the detection of the mark is dependant of the Mpeg-4 format.

Piva et al developed an additive embedding scheme in the wavelet domain for Mpeg-4 video streams [5]. The detection of the mark requires the decompression of the video and is invariant to format conversion. The scheme is robust to object translation and VOL modification. It is important to point out that these different schemes are not robust to object manipulations such as scaling operations or rotations because in these cases the synchronization of the mark cannot be achieved.

The proposed scheme has been developed to enable signature detection after object manipulations. We focus our works on providing an automatic watermarking scheme able to orientate and scale the signature according to the object shape. Contrary to still image watermarking schemes robust to geometric transformations by embedding an artificial information to retrieve the transform [6][7], our approach is to bound the signature with the object content. Such a scheme belongs to second generation watermarking schemes [8][9] where the video content is used to enable the synchronization of the mark.

\subsection{Signature orientation}

To detect the signature and decode the embedded message after VO rotations, we have decided to orientate the signa- 
ture according the principal direction of the object shape. We decided to employ Principal Component Analysis to reach such objective and obtain the principal directions $\vec{U}$ and $\vec{V}$ of the VOP[10] (vectors $\vec{U}$ and $\vec{V}$ denotes directions providing maximal variance of the data). In this context, the set of data is represented by a set of 2-D random variables $m_{i}\left(x_{i}, y_{i}\right)$ that belong to the object mask $M . \vec{U}$ and $\vec{V}$ represent consequently the eigenvectors of the $2 \times 2$ covariance matrix $C(O)$.

The main direction of the VOP, denoted $\alpha_{C P A}$, represents the angle between the eigenvector $\vec{U}$ and the horizontal direction of the object plan, denoted $\vec{i}$ :

$$
\alpha_{C P A}=(\vec{i} ; \vec{U}) \bmod (\pi)
$$

Such a process permits to obtain a sharp orientation of the video object. Tabular 1 illustrates angle variation in degrees for different objects rotations of 40 degree multiples on the "Bream" VO. Results indicate the fact that the presented estimator is extremely accurate (mean precision is over $10^{-2}$ degree).

\begin{tabular}{|l|l|l|l|l|l|}
\hline angle & 0 & 40 & 80 & 120 & 160 \\
\hline error & 0 & 0.005 & 0.007 & 0.020 & 0.007 \\
\hline
\end{tabular}

Table 1. Orientation evaluations in degree of VO "Bream" using PCA.

\subsection{Signature scaling}

To enable the detection of the mark after a VOP scaling operation, we extract a specific value of the VOP scale from the PCA analysis.

Supposing that the VOP, denoted $O$, has been scaled by a factor $s$, the value $\Gamma(O)$ represents a real proportional to the VOP scale. Given:

$$
\Gamma(O)=\left(\lambda_{1}(O)+\lambda_{2}(O)\right)^{\frac{1}{4}}
$$

We obtain:

$$
\frac{\Gamma(s O)}{\Gamma(O)}=\frac{\left(\lambda_{1}(s O)+\lambda_{2}(s O)\right)^{\frac{1}{4}}}{\left(\lambda_{1}(O)+\lambda_{2}(O)\right)^{\frac{1}{4}}}=s
$$

Table 2 illustrates the capacity of the proposed method to estimate the specific scale of the VOP. Our tests were applied on the "Akiyo" VO for scale factors varying from 1 to 0.2 . In all configurations, the estimation error is under $10^{-2}$ and consequently this scale estimator is reliable.

\section{EMBEDDING SCHEME}

Our embedding scheme can be considered as an additive scheme with the particularity that the signature is inserted

\begin{tabular}{|l|l|l|l|l|l|}
\hline$s(\%)$ & 1 & 0.8 & 0.6 & 0.4 & 0.2 \\
\hline$\Gamma(s O)$ & 2.616 & 2.089 & 1.569 & 1.041 & 0.522 \\
\hline error & 0 & 0.003 & 0.000 & 0.005 & 0.001 \\
\hline
\end{tabular}

Table 2. Scaling evaluation of "Akiyo" VO using PCA.

in an appropriate referential depending on the object shape. This scheme can be decomposed in six different step illustrated on Figure 1:

1. As presented in the previous section, a principal component analysis is performed on the VO binary mask $O$. Values of $\alpha_{C P A}$ and $\Gamma$ are extracted.

2. A random sequence (denoted $S$ ), composed of a set of $\{+1,-1\}$ spread on $2 \times 2$ blocs, is generated from a secret key $K$.

3. $S$ is thereafter scaled and rotated using $\alpha_{C P A}$ and $\Gamma$ to obtain a sequence $S^{\prime}$. Each coordinate $\left(x^{\prime}, y^{\prime}\right)$ are given by:

$$
\begin{gathered}
{\left[\begin{array}{l}
x^{\prime} \\
y^{\prime}
\end{array}\right]=\Gamma\left[\begin{array}{rr}
\cos \alpha & -\sin \alpha \\
\sin \alpha & \cos \alpha
\end{array}\right]\left[\begin{array}{l}
x-x_{c} \\
y-y_{c}
\end{array}\right]} \\
+\Gamma\left[\begin{array}{l}
x_{c} \\
y_{c}
\end{array}\right]=\Gamma\left(\mathbf{R}(\alpha)\left[\begin{array}{l}
x-x_{c} \\
y-y_{c}
\end{array}\right]+\left[\begin{array}{l}
x_{c} \\
y_{c}
\end{array}\right]\right)
\end{gathered}
$$

where $\left(x_{c} ; y_{c}\right)$ are the coordinate of the centre of gravity of the VOP shape.

We after proceed to an interpolation process using a spine-cubic convolution kernel to preserve the high frequencies of $S$.

4. $S^{\prime}$ is thereafter weighted by a visual mask depending of VOP luminance to obtain the sequence $S_{v}^{\prime}$. We exploit the fact that the human eye is less sensitive to contrast in dark or bright areas than to middle luminance areas.

5. $S_{v}^{\prime}$ is finaly added to the object texture to create the marked object.

\section{DETECTION SCHEME}

As in classical additive schemes, the detection of the mark is performed using a correlation criterion. The detection process can be divided in five steps illustrated on figure 2 .

1. As in the embedding scheme, one sequence $S$ is generated using a secret key $K$.

2. We transform the object texture to obtain an object $O_{m}$ in such a way that $O_{m}$ orientation is null and $O_{m}$ 


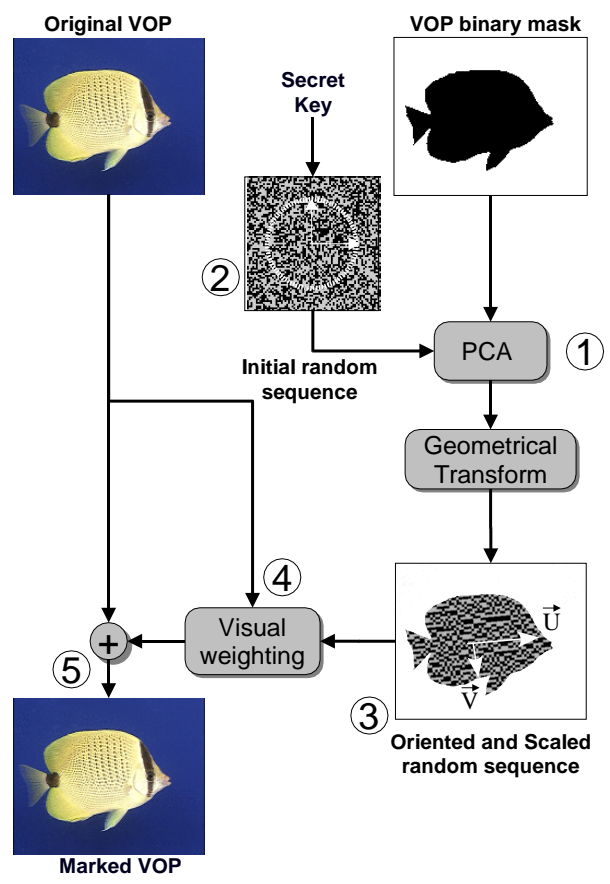

Fig. 1. Signature embedding scheme.

scale is equal to 1 . This operation is equivalent to invert equation 1 :

$$
\left[\begin{array}{l}
x \\
y
\end{array}\right]=\Gamma^{-1}\left(\mathbf{R}(-\alpha)\left[\begin{array}{l}
x^{\prime}-x_{c}^{\prime} \\
y^{\prime}-y_{c}^{\prime}
\end{array}\right]+\left[\begin{array}{l}
x_{c}^{\prime} \\
y_{c}^{\prime}
\end{array}\right]\right)
$$

3. A Wiener prediction is applied on $O_{m}$ to minimize the texture contribution of the original object before the correlation computation [11].

4. The correlation value corr between $\hat{W}$ and the random sequence $W$ is evaluated::

$$
\operatorname{corr}=\sum_{(i, j) \in O} \hat{W}(i, j) W(i, j)
$$

5. The corr value is stoked in an accumulation variable $\operatorname{corr}_{A}$ initialised to $0 . \operatorname{corr}_{A}$ is thereafter compared with a threshold depending on a false-alarm probability $\lambda\left(p_{f a}\right)$. In the case of $\operatorname{corr}_{A}>\lambda\left(p_{f a}\right)$, the signature is consequently detected inside the VOP.

\section{RESULTS}

We have tested the presented method on the Bream sequence, composed of 300 frames in a CIF format $(352 \times 288)$ using a 4:2:0 sampling. Only the luminance components of the different frames are marked. The Bream sequence is composed of two video objects, a fish denoted $V O_{11}$ delimited

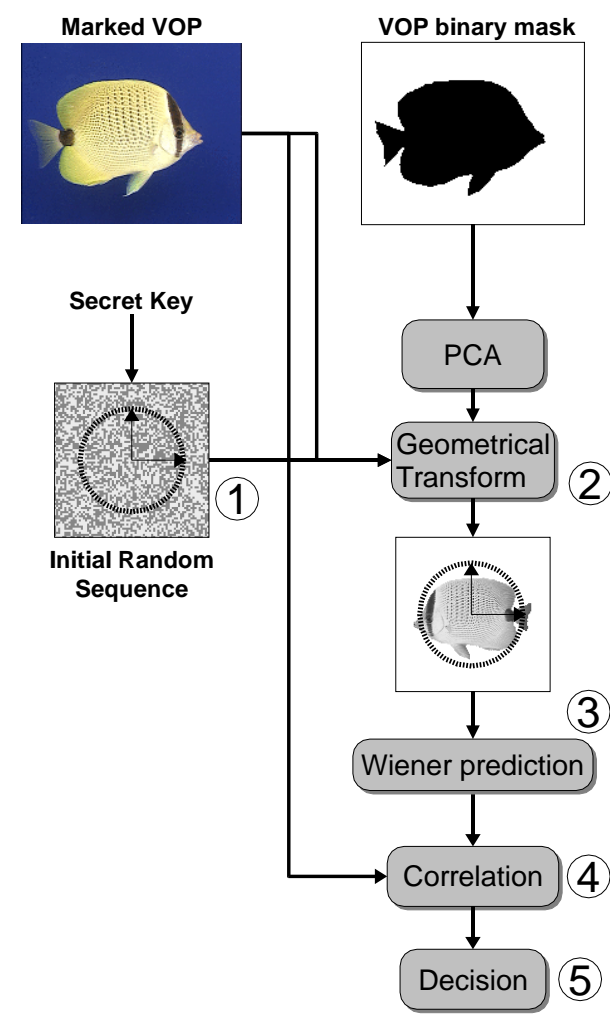

Fig. 2. Signature detection and decoding scheme.

by a $272 \times 185$ bounding box and a background representing the aquarium denoted $V O_{12}$. Only $V O_{12}$ is marked in theses tests. The final decision is determined by the sum of correlations obtained for each sequence VOP. The evaluation of the proposed scheme was performed for different VO manipulations and different bite-rates. The bite-rates have been chosen to represent a wide variety of multimedia applications. $1 \mathrm{Mb} / \mathrm{sec}, 800 \mathrm{~Kb} / \mathrm{sec}, 600 \mathrm{~Kb} / \mathrm{sec}, 400 \mathrm{~Kb} / \mathrm{sec}$ and $200 \mathrm{~Kb} / \mathrm{sec}$ have been used. The marked sequence does not have noticeable differences from the original one, even in its uncompressed version.

The VO manipulations that have been tested are:

- VO scaling of $40 \%$

- VO rotation of 20 degrees

- VO mask sub-sampling with a factor equal to 8 pixels, this is commonly used to perform lossy compression of the VO shape.

We have performed a statistical analysis of $\operatorname{corr}_{A}$ to determine a false-alarm threshold. In these tests the false-alarm probability was fixed to $10^{-7}$. The different correlation values are sum-up on Fig. 4. Theses results illustrate the fact that our detection scheme is robust to bit-rate reduction for very low bit-rates. But the most important property 


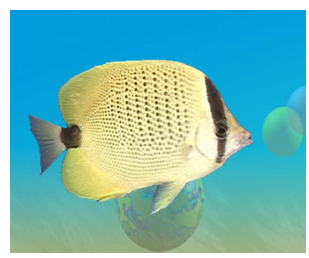

original sequence

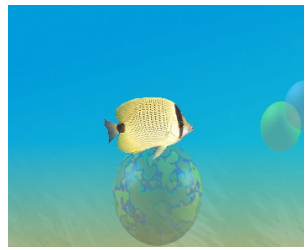

scaled sequence

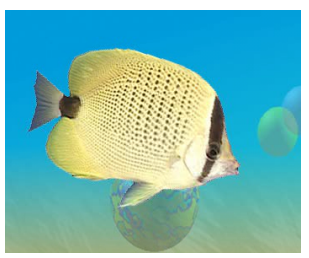

rotated sequence

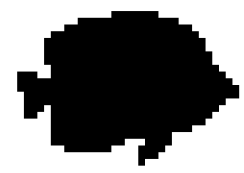

sub-sampling
Fig. 3. Evaluated configurations.

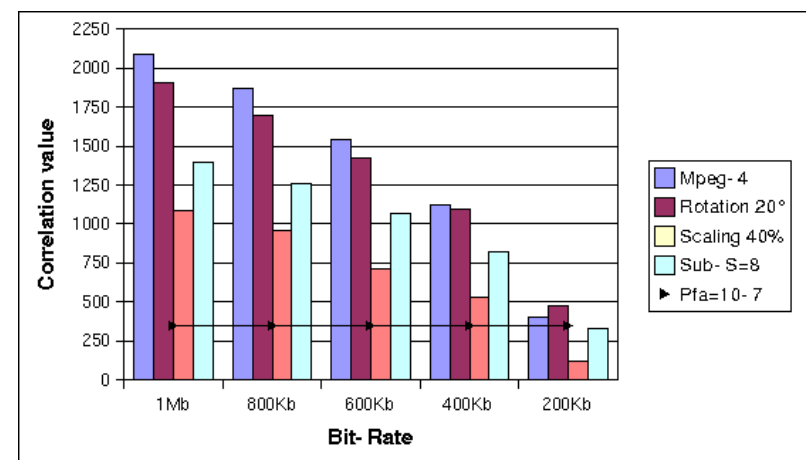

Fig. 4. Signature detection results for different bit-rate.

of the presented method is his robustness to scaling of rotation operations for different bit-rates. Finally the mask sub-sampling operation by a factor equal to 8 seems to not affect the detection process in an important way. In our futur works we will concentrate the tests on real new segmentation conditions.

\section{CONCLUDING REMARKS}

The main idea of the presented paper is the use of Principal Component Analysis tools to perform signature detection after Video Object manipulations. VO boundary analysis permits to obtain orientation and scale information of the VO. We have used this information to create a additive scheme having low computational complexity that permits the detection of the mark after geometrical manipulations or shape modifications. Our future works will be devoted to develop embedding in the compressed domain and test real segmentation conditions.

\section{REFERENCES}

[1] T. Ebrahimi and C. Horne, "Mpeg-4 natural video coding - an overview," Signal Processing, vol. 15(4-5), pp. 365-385, 2000.

[2] F. Hartung and M. Kutter, "Multimedia watermarking techniques," Proceedings of the IEEE, vol. 87, no. 7, pp. 1079-1107, july 1999.

[3] Jack Lacy, Niels Rump, and Panos Kudumakis, "Mpeg-4 intellectual property management \& protection (ipmp) overview \& applications document," Tech. Rep., MPEG-4, Dec. 1998.

[4] M. Barni, F. Bartolini, V. Capelini, and N. Checcacci, "Object watermarking for mpeg-4 video streams copyright protection," in EI'2000: Security and Watermarking of Multimedia Content II, San Jose, California USA, 23-28jan 2000, vol. 3971 of SPIE Proceedings, pp. 465-476.

[5] A. Piva, R. Caldelli, and A. De Rosa, "A dwt-based object watermarking system for the mpeg-4 video streams," in IEEE-ICIP'00, Vancouver, Canada, Sept. 2000, pp. 5-9.

[6] Shelby Pereira and Thierry Pun, "Fast robust template matching for affine resistant image watermarking," in International Workshop on Information Hiding, Dresden, Germany, 29 September -1 October 1999, vol. LNCS 1768 of Lecture Notes in Computer Science, pp. 200-210, Springer Verlag.

[7] S. Voloshynovskiy, A. Herrigel, and Y. B. Rytsar, "Watermark template attack," in EI'2001: Security and Watermarking of Multimedia Content III, Ping Wah Wong and Edward J. Delp, Eds., San Jose, California USA, 22-25jan 2001, SPIE Proceedings.

[8] M. Kutter, S. K. Bhattacharjee, and T. Ebrahimi, "Towards second generation watermarking schemes," in IEEE-ICIP'99, Kobe (Japan), Oct. 1999, vol. I, pp. 320-323.

[9] P. Bas, J-M Chassery, and B. Macq, "Image watermarking: An evolution to content based approaches," Pattern Recognition, Special Issue on Image/Video Communication edited by D. Aboutajdine, to be published, 2001.

[10] R. C. Gonzalez and P. Wintz, Digital Image Processing, Addison Wesley, 1987.

[11] J. R. Hernandez and F. Perez-Gonzalez, "Statistical analysis of watermarking schemes for copyright protection of images," Proceedings of the IEEE, vol. 87, no. 7 , pp. 1142-1143, july 1999. 